\title{
Skin innate immune system in psoriasis: friend or foe?
}

\author{
Brian J. Nickoloff \\ Department of Pathology and Department of Microbiology and Immunology, \\ Loyola University of Chicago, Cardinal Bernardin Cancer Center, 2160 South First Avenue, Room 301, \\ Maywood, Illinois 60153-5385, USA. Phone: (708) 327-3241; Fax: (708) 327-3239; E-mail: Bnickol@luc.edu.
}

Immunobiological and genetic approaches to understand psoriasis are rapidly converging. Psoriasis is a common inherited disease that has been described for 2000 years, and affects $2-3 \%$ of the world-wide population. Immunologists, cell biologists, and molecular geneticists are becoming increasingly preoccupied with the cellular and molecular events that produce the commotion in the epidermis that results in a psoriatic plaque. Dermatologists familiar with this enigmatic and chronic disorder recognize that emergence of the distinctive phenotype (widely scattered erythematous plaques; Figure 1) is likely to be multifactorial, related to both environmental and genetic triggering factors (1). Psoriasis currently tops the list, based on population frequencies and familial clustering, of human autoimmune and immunerelated diseases (2).

Despite its accessibility, frequency, and persistence since antiquity, many puzzling questions about psoriasis remain unanswered. Perhaps the most obvious unresolved issue, reflecting the lack of an identification of the major genetic susceptibility determinants, is whether this disorder fundamentally reflects an abnormality in the epidermal keratinocyte $(\mathrm{KC})$ or bone-marrow derived

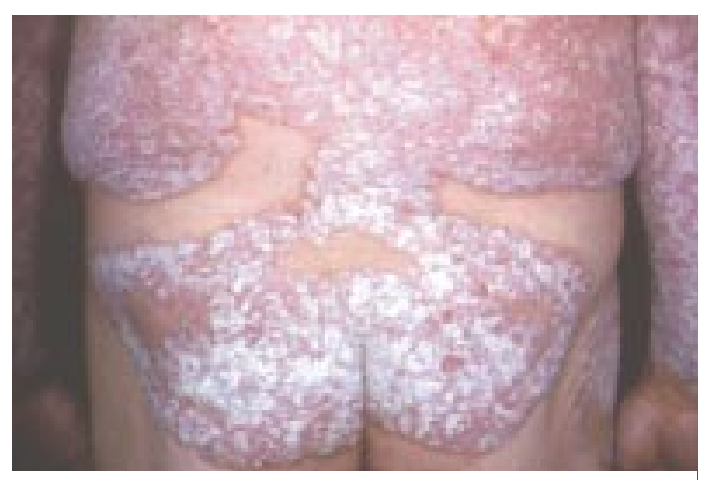

Figure 1

Clinical photograph of extensive psoriatic plaque formation with protective shield-like appearance encircling the trunk, arms, and lower back/buttocks. immunocyte. This distinction becomes blurred because of cross-talk between activated KCs and immunocytes that begins immediately upon lesion formation and culminates in the mature psoriatic plaque. This uncertainty prompts further questions: Does psoriasis represent a primary defect in the terminal differentiation response to injury by KCs that fail to produce a normal stratum corneum and, therefore, do not create a protective physiological barrier? Alternatively, is the aberrant $\mathrm{KC}$ differentiation program in psoriasis a consequence of an influx of pathogenic immunocytes participating in a rogue auto-immune reaction? These possibilities are relevant to our understanding of the hyperresponsiveness of pre-psoriatic skin reported by Travers et al. in this issue of the JCI (3). In this Commentary, I synthesize available data into a multistep model, taking a novel view of psoriasis from the perspective of the innate immune system. This approach may lead to new avenues for investigators confronting this perplexing skin problem that involves dynamic and reversible epidermal remodeling.

\section{Role of innate immunity in adaptive response of skin}

Since few other organ systems are as constantly confronted by potentially life-threatening infectious agents, it should not be surprising that many cutaneous protective strategies have evolved involving both innate and acquired immunity. The primary intrinsic anti-infectious protective shield of the skin is the stratum corneum, a non-viable surface layer of "mummified" anucleated cells derived from terminally differentiated KCs. The stratum corneum consists of highly cross-linked proteinaceous cellular en- velopes with extracellular lipid lamellae, consisting mainly of ceramides, free fatty acids, and cholesterol (4). This biological "Saran Wrap" can be removed by repeated cellophane tape stripping to perturb the barrier function (Figure 2), altering transepidermal water loss and calcium ion gradients. Such mild trauma renders the skin permeable to infectious agents and/or their secreted products such as bacteria-derived super-antigens (SAg's). One of the immediate protective responses of skin to barrier perturbation is release of lamellar bodies (LB) containing pre-formed lipid and hydrolytic enzymes, as well as cytokines such as TNF- $\alpha$ and IL- $1(5,6)$. Other early events include influx of neutrophils that release anti-microbial agents, production of anti-bacterial defensins by KCs, and enhanced desquamation of the stratum corneum itself (7). In healthy individuals, these innate responses quickly restore cutaneous homeostasis, but in genetically predisposed patients with psoriasis, new lesions are created that can persist for decades. Because of the aberrant epidermal differentiation program in such lesions, psoriatic plaques lack a normal granular cell layer or intact stratum corneum (8). Despite this compromised structural shield, however, the fullydeveloped psoriatic plaque is resistant to invasion by infectious organisms (9).

One possible explanation for this resistance to infection involves a compensatory response by members of the cellular immune system, particularly $\mathrm{T}$ cells expressing natural killer receptors (NKRs). There are 3 principle reasons for considering immunocytes in the pathogenesis of psoriasis. First, many different types of barrier defects in the epidermis have been produced in transgenic mice that do not produce a psoriatic plaque. For example, in keratin-10 knockout mice, although hyperkeratosis occurs as the animals mature, other stigmata of psoriasis, such as loss of the 


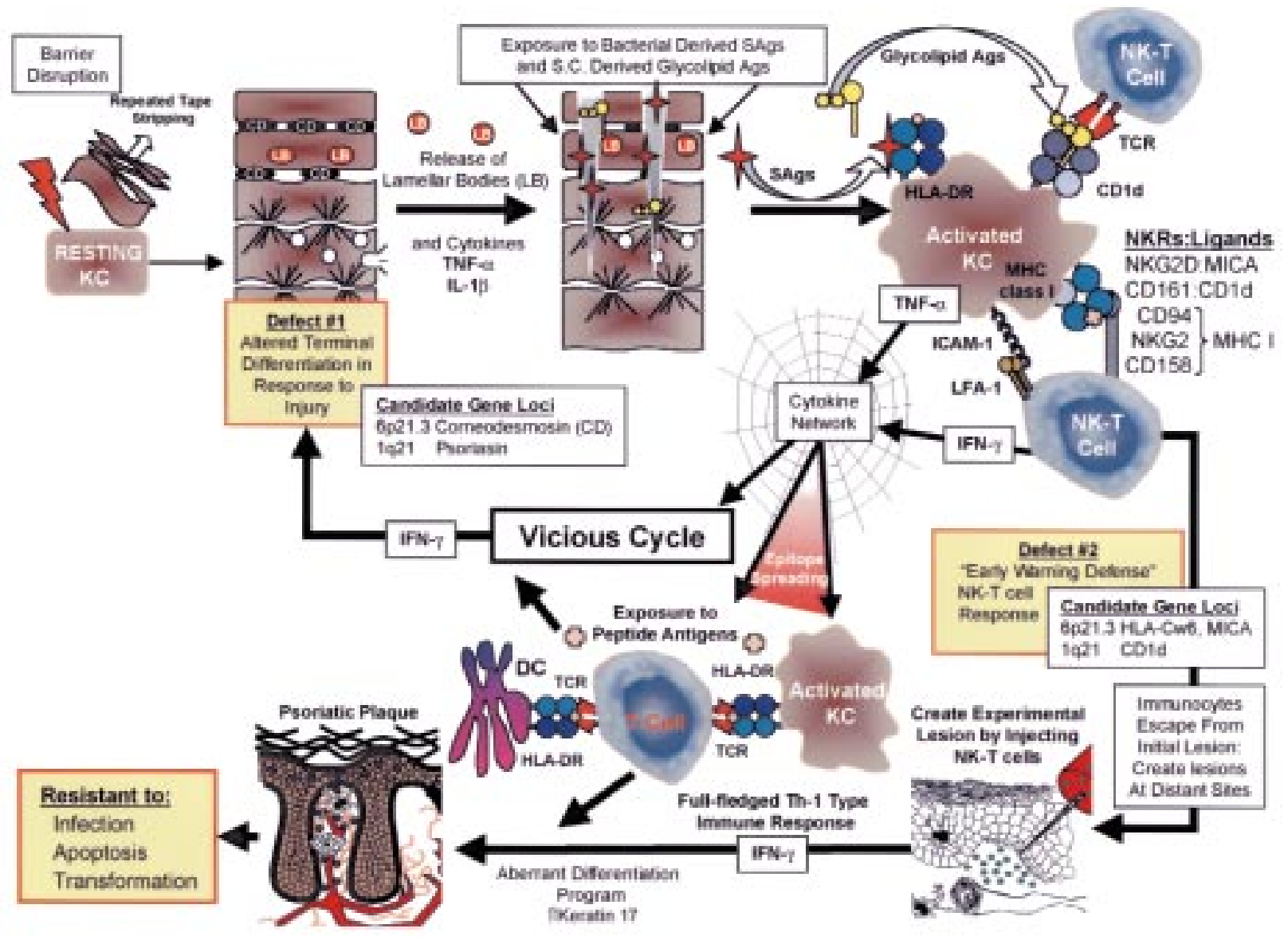

Figure 2

Schematic representation depicting how a confederacy of innate immunity-based genes and cells may conspire to create a psoriatic lesion. Whether triggered by an exogenous event that disrupts KC barrier function (with or without bacterial infection) or by an endogenously-derived infiltration of activated immunocytes, the final mature psoriatic plaque includes an epidermal compartment resistant to infections, apoptosis, and transformation. The complexity of interactions between innate immunity and acquired immunity in skin is depicted as a multi-step model highlighting 2 key abnormalities in psoriasis, including (a) defective terminal differentiation/barrier function and (b) hyperreactivity of the skin immune system including T cells bearing NKRs, which can evolve into a full-fledged Th1-type cell-mediated reaction involving adaptive immune components.

granular cell layer, elongation of rete pegs, or an angiogenic tissue reaction, are not present (10). Thus, as current dogma now suggests, the barrier defect in psoriasis more likely represents a consequence, rather than a cause, of infiltration by activated immunocytes that release cytokines that trigger an aberrant differentiation program in the epidermis (11). Second, many drugs that selectively target activated $T$ cells are very effective in producing remissions in psoriasis patients (12). Third, after an upper respiratory infection, psoriatic patients will develop numerous skin lesions that do not represent primary cutaneous infections. As discussed below, this finding suggests that activated immunocytes responsive to the initial bacterial infection can exit the pharynx and migrate to skin where they produce Th1-type cytokines (11).
Similarly, transgenic mice that constitutively produce gamma interferon (IFN- $\gamma$ ) in the epidermis, display profound barrier defects in the skin (13); intradermal injection of IFN- $\gamma$ can also trigger psoriatic lesions in genetically susceptible patients (14). Interestingly, the altered keratin profile seen in keratin 10 knockout mice with disrupted barrier includes aberrant expression of keratin 17 , which is also characteristically present in psoriatic plaques (15) and can be induced by exposure to IFN$\gamma$ either in-vivo (13) or in-vitro (16).

\section{The innate immunity-based model of psoriasis}

Given the prevalence of psoriasis worldwide there may be overlooked beneficial attributes for this genotype. There are many examples in which genetic abnormalities are linked to pathogen resistance
(17). While it would be difficult to convince most patients with psoriasis that their disease is actually a blessing rather than a burden, it does appear that formation of a psoriatic plaque provides a rather impressive protective shield for the skin. Because it is clear that the barrier function of normal skin can be acutely breached or, as in psoriasis, chronically altered, we can ask what secondary hostresponse follows this epidermal defect. Innate immune-based reactions suggest a back-up system distinct from the aforementioned KC-and neutrophil-based responses. Recently, we uncovered in skin a previously overlooked subset of $\mathrm{T}$ cells that express surface receptors typically present on natural killer (NK) cells (18, 19). These receptors (NKRs) are on lymphocytes that also express T-cell receptors (TCRs), and such cells (i.e., NK-T cells) may represent a link between the 
innate (via NKRs) and acquired (via TCRs) immune systems. Not only do acute and chronic psoriatic plaques contain NKR-bearing T cells, but such cells can be triggered to produce high levels of IFN- $\gamma$ (within 1 to 2 days) when co-cultured with CD1d-positive KCs (unpublished observation). It should be noted that certain glycolipids, such as $\alpha$-galactosylceramide, enhance the CD1d-related NK-T-cell production of IFN- $\gamma$. Upon barrier disruption, it is likely that bacterial SAg's reach into the mid- and lower epidermis (2) and that a variety of glycolipids derived from stratum corneum also become admixed with the subjacent cytokine-activated KCs. It is conceivable that production of IFN- $\gamma$ by CD1drestricted, glycolipid-responsive NK-T cells could synergize with the KC-derived TNF- $\alpha$ to create a cytokine network that facilitates elimination of the invading organisms once the epidermal barrier defect has been created (20). In addition, glycolipid antigens unveiled during lesion formation could further fuel NK$\mathrm{T}$ cell interaction with KCs, thereby creating a vicious cycle (Figure 2).

The essence of this new model for psoriasis is that the compensatory immune response mediated by NK-T cells is coupled to the defective barrier function. The localized NK-T cell response in skin can be viewed as an "early warning"-type back-up system, prone to overreacting to the altered barrier, even in the absence of a local infection. Although this cutaneous hyperresponsiveness may have been of benefit in pre-antibiotic centuries, its useful- ness in modern society is questionable. Because of this response, lesions can multiply when locally activated NK-T cells escape the initial site and spread to non-lesional skin. I would suggest that the appearance of skin lesions following bacterial pharyngitis is due to the ability of SAg not only to induce skin homing receptors by $\mathrm{T}$ cells (21) but also to induce NKR expression (22). Thus, once NKR-bearing $T$ cells reach a critical threshold in the bloodstream, they can enter the skin and, upon contact with CD1d-bearing KCs, upregulate production of IFN- $\gamma$, a cytokine that triggers an aberrant differentiation program in the epidermis. This "insideout" creation of a new psoriatic lesion would proceed following the same cytokine cascade that results from physical disruption of the stratum corneum. Thus, defective barrier formation by KCs and an exaggerated NK$\mathrm{T}$ cell response conspire to produce a psoriatic plaque, which solves the primordial problem of controlling the spread of infectious pathogens.

\section{Therapeutic intervention options in psoriasis}

Based on this multistep model, one can envision selectively targeting specific stimuli and host responses involved in the pathogenesis of psoriasis (Figure 3). As early inflammatory-based events involving innate immunity may affect more distal events highlighting acquired immunity with T-cell activation (23), the precise timing of the therapeutic intervention becomes important in treating early, evolving lesions rather than established plaques. In contrast to the use of non-specific immunomodulatory agents the emphasis based on this model is to develop targeted methods to reverse the hyperresponsiveness of psoriatic skin. Therapies to influence barrier function might include drugs that promote terminal differentiation even in the presence of cytokines such as TNF- $\alpha$ and IFN- $\gamma$ (i.e., peroxisome proliferator-activated receptors; PPAR activators) (24). To block either NK-T-cell or conventional Tcell activation, and hence to reduce IFN$\gamma$ production, it may be possible to block TCR-based or NKR-based signaling using specific mAb's, or to interfere with additional costimulatory molecules using CTLA4Ig (25) or anti-LFA-1/ICAM1 mAb's (26). Finally, blocking or interrupting the angiogenic switch, particularly involving vascular endothelial growth factor-mediated (VEGF-mediated) neovascularization, represents another therapeutic opportunity in psoriasis.

\section{Future direction and challenges}

Clearly an incontrovertible view of psoriasis will emerge by correctly identifying all genetic mutations and by elucidation of the molecular pathway responsible for masterminding this multicellular conspiracy in skin. While several candidate gene loci have been proposed in psoriasis (9), only 2 regions will be discussed. One region is within the MHC complex on 6p21.3 (27) and includes the non-HLA gene-encoding corneodesmosin (CD) a protein with homology to keratin-10 $(28,29)$. The other region includes a cluster of genes on chromosome 1q21 (30). Potential candidate genes encode markers of epidermal differentiation such as corneodesmosin, psoriasin, and CD1d, to name a few $(18,30,31)$. It should be noted that corneodesmosin was not initially considered to have an immunological function (and

\section{Figure 3}

Potential means to reverse the hyperresponsiveness of psoriatic skin. Possible intervention points based on a multistep model of psoriasis, beginning with inflammatory-type reactions involving innate immunity and culminating in acquired immunity and activation of an angiogenic switch. 
hence perhaps was misplaced in the genome amidst other important immune responsive genes including the MHC class I and II antigens, complement proteins, and potent immunomodulatory cytokines), but its location at $6 \mathrm{p} 21$ may reflect its role in formation of stratum corneum - the primary immunological defensive shield for the entire body.

Besides these genetic approaches, it is clear that more work is required to better understand how innate immunity can affect adaptive immune responses $(32,33)$ and to unravel the basis of the hyperresponsiveness of pre-psoriatic skin. It will be of critical importance to compare phenotypic profiles and function of relevant ligand/receptors mediating activation of $\mathrm{T}$ cells bearing NKRs between individuals with and without psoriasis. Molecular pairs of interest include NKRs interacting with class I MHC alleles (i.e., Cw6): CD158, MICA (NKG2D), CD1d reactive TCR/ costimulatory molecule CD161, and CD94/NKG2 receptors for HLA-Ebound peptides (12). An intriguing line of investigation will be to determine if patients have mutations that prompt an exaggerated or dysregulated interaction between NK-T cells and epidermis. It is also possible that, besides involvement of glycolipids and lectinlike NKRs on these $\mathrm{T}$ cells, there are also peptide-bound antigens in stratum corneum which contribute to persistence and/or evolution of initial lesions into conventional full-fledged $\mathrm{T}$ cell-mediated reactions (34). Epitope spreading involving other self-antigens including peptide antigens that participate in enhanced immune responses may also be considered in chronic $\mathrm{T}$ cell-mediated dermatitis. In addition to resisting infection, plaque KCs resist apoptosis as one aspect of serving as a protective shield (12). Plaques also resist transformation despite exposure to initiators and promoters such as anthralin, crude coal tar, UV-light, and chronic immune suppression (35). Taken together, the KCs in psoriatic plaques follow an aberrant differentiation pathway and resist apoptosis and transformation, features that resemble the state of cellular senescence (12).

In summary, psoriasis is a complex and highly dynamic disease process likely to continue to challenge our scientific approaches and imagination.
Even though many dermatologists continue to treat psoriasis with relatively nonspecific immunosuppressive drugs, I hope this Commentary prompts exploration of new therapeutic approaches and rethinking regarding the role of innate versus acquired immunity in the skin. In any event, the aforementioned observations suggest that we should broaden our viewpoint as to what constitutes innate immunity in skin and its relevance to psoriasis.

\section{Acknowledgments}

Many thanks to members of the Loyola University Skin Cancer Program for helpful discussions; Heide Bauer, Crystal Tabor, and Brian Bonish for preparation of figures; and Theresa Hermann for manuscript preparation. June Robinson supplied the clinical photograph. Craig Thompson reviewed the manuscript, and John Ashkenas provided valuable criticism. The author acknowledges the National Institutes of Health for 15 years of grant support related to psoriasis.

1. Farber, E.M., and Nall, L.M. 1974. The natural history of psoriasis in 5600 patients. Dermatologica. 148:1-18.

2. Vyse, T.J., and Todd, J.A. 1996. Genetic analysis of autoimmune disease. Cell. 85:311-318.

3. Travers, J.B., et al. 1999. Epidermal HLA-DR and the enhancement of cutaneous reactivity to superantigenic toxins in psoriasis. J. Clin. Invest. 104:1181-1189.

4. Elias, P.M., et al. 1993. Normal mechanisms and pathophysiology of epidermal permeability barrier homeostasis. Curr. Opin. Dermatol. 231-237.

5. Tsai, J.-C., et al. 1996. Permeability barrier disruption alters the localization and expression of TNF-alpha protein in the epidermis. Arch. Dermatol. Res. 286:242-248.

6. Nickoloff, B.J., and Naidu, Y. 1994. Perturbation of epidermal barrier function correlates with initiation of cytokine cascade in human skin. J. Am. Acad. Dermatol. 30:535-546.

7. Harder, J., Bartels, J., Christophers, E., and Schroeder, J.M. 1997. A peptide antibiotic from human skin. Nature. 387:861.

8. Ghadially, R., Reed, J.T., and Elias, P.M. 1996. Stratum corneum structure and function correlates with phenotype in psoriasis. J. Invest. Dermatol. 107:558-564.

9. Henseler, T. 1997. The genetics of psoriasis. J. Am. Acad. Dermatol. 37:s1-s11.

10. Porter, R.M., et al. 1996. Gene targeting at the mouse cytokeratin 10 locus: severe skin fragility and changes of cytokeratin expression in the epidermis. J. Cell. Biol. 132:925-936.

11. Valdimarsson, H., et al. 1995. Psoriasis: a T-cell mediated autoimmune disease induced by streptococcal superantigens. Immunol. Today. 16:145-149.

12. Nickoloff, B.J. 1999. The immunological and genetic basis of psoriasis. Arch. Dermatol. 135:1104-1110.

13. Carroll, J.M., et al. 1997. Transgenic mice expressing IFN- $\gamma$ in the epidermis have eczema, hair hypopigmentation, and hair loss. J. Invest. Dermatol. 108:412-422.

14. Fierlbeck, G., et al. 1990. Psoriasis induced at the injection site of recombinant interferon gamma.
Results of immunohistologic investigations. Arch. Dermatol. 126:351-355.

15. DeJong, F.M.G.J., et al. 1991. Keratin 17: a useful marker in anti-psoriatic therapies. Arch. Dermatol. Res. 283:480-482.

16. Vogel, U., et al. 1995. Transcriptional activation of psoriasis-associated cytokeratin 17 by interferon-- $\gamma$. Analysis of $\gamma$-interferon activation sites. Eur. J. Biochem. 227:143-149.

17. Olson, M.V. 1999. Molecular evolution ' 99 . When less is more: gene loss as an engine of evolutionary change. Am. J. Hum. Genet. 64:18-23.

18. Nickoloff, et al. 1999. Response of murine and normal human skin to injection of allogeneic blood-derived psoriatic immunocytes: detection of $\mathrm{T}$ cells expressing receptors typically present on natural killer cells including CD94, CD158, and CD161. Arch. Dermatol. 135:546-552.

19. Nickoloff, B.J., and Wrone-Smith, T., 1999. Injection of pre-psoriatic skin with CD4+ $\mathrm{T}$ cells induces psoriasis. Am. J. Pathol. 155:145-158.

20. Barker, J.N.W.N., et al. 1991. Keratinocytes as initiators of inflammation. Lancet. 337:211-214.

21. Leung, D.Y.M., et al. 1995. Bacterial superantigens induce $T$ cell expression of the skin-selection homing receptor: the cutaneous lymphocyteassociated antigen via stimulation of interleukin 12 production. J. Exp. Med. 181:747-753.

22. Mingari, M.-C., et al. 1998. HLA class I-specific inhibitory receptors in human $\mathrm{T}$ lymphocytes: interleukin 15-induced expression of CD94/ NKG2A in superantigen-activated CD8+ T cells. Proc. Natl. Acad. Sci. USA. 95:1172-1177.

23. Vella, A.T., McCormack, J.E., Linsley, P.S., Kappler, J.W., and Marrack, P. 1995. Lipopolysaccharide interferes with the induction of peripheral $\mathrm{T}$ cell death. Immunity. 2:261-270.

24. Vamecq, J., and Latruffe, N. 1999. Medical significance of peroxisome proliferator-activated receptors. Lancet. 354:141-148.

25. Abrams, J.R., et al. 1999. CTLA4Ig-mediated blockade of T-cell costimulation in patients with psoriasis vulgaris. J. Clin. Invest. 103:1243-1252.

26. Nickoloff, B.J. 1998. Pathogenesis and immunointervention strategies in psoriasis. Mol. Med. Today. 4:512-513.

27. Trembath, R.C., et al. 1997. Identification of a major susceptibility locus on chromosome $6 \mathrm{p}$ and evidence for further disease loci revealed by a two stage genome-wide search in psoriasis. Hum. Mol. Genet. 6:813-820.

28. Zhou, Y., and Chaplin, D.D. 1993. Identification in the HLA class I region of a gene expressed late in keratinocyte differentiation. Proc. Natl. Acad. Sci. USA. 90:9470-9474.

29. Allen, M.H., et al. 1999. A non-HLA gene within the MHC in psoriasis. Lancet. 353:1589-1590.

30. Hardas, D.B., et al. 1996. Assignment of psoriasin to human chromosomal band 1q21: coordinate overexpression of clustered genes in psoriasis. $J$. Invest. Dermatol. 106:753-758.

31. Capon, F., et al. 1999. Searching for psoriasis susceptibility genes in Italy: genome scan and evidence for a new locus on chromosome 1. J. Invest. Dermatol. 112:32-35.

32. Fearon, D.T., and Locksley, R.M. 1996. The instructive role of innate immunity in the acquired immune response. Science. 272:50-54.

33. Metzhitov, R., and Janeway, C.A., Jr. 1997. Innate immunity: impact on the adaptive immune response. Curr. Opin. Immunol. 9:4-9.

34. Hales, J.M., and Camp, R.D.R. 1999. T cell stimulating stratum corneum antigens: characterization by chromatography and electrophoresis indicates limited diversity. J. Invest. Dermatol. 113:355-363.

35. Lindelof, B., Eklund, G., Liden, S., and Stern, R.S. 1990. The prevalence of malignant tumors in patients with psoriasis. J. Am. Acad. Dermatol. 22:1056-1060. 